\title{
EDITORIAL
}

\section{POLÍTICAS DE SALUD: FUNDAMENTOS Y COSTE-EFECTIVIDAD}

\section{Vicente Ortún $(1,2)$}

(1) Profesor del Departamento de Economía y Empresa y Director del Centro de Investigación en Economía y Salud, Universidad Pompeu Fabra, Barcelona.

(2) Visitante del Departamento de Economía del MIT, Cambridge, Massachusetts.

Los artículos que, en respuesta a la convocatoria de la RESP, componen este Monográfico reflejan un enfoque de la Economía de la Salud orientado a la investigación y resolución de problemas de salud y servicios sanitarios, con sus practicantes repartidos entre departamentos de economía y empresa, de ciencias de la salud, escuelas de salud pública, organizaciones sanitarias, etc. En diferente grado, todos tratan de reducir la brecha, ya señalada en España $^{1}$, entre presencia e influencia de la Economía de la Salud utilizando como mecanismo retórico la publicación en una revista de salud pública.

La preocupación por la mejora de la efectividad de las políticas de salud así como los fundamentos de las mismas constituyen la urdimbre compartida por los trabajos que se recogen en el Monográfico (de ahí el título del editorial). Con la elocuencia de sus resultados y la solidez de sus prescripciones para la acción se pretende contribuir a un mínimo consenso científico. Lejos quedará que los cursos recomendados de acción (policy) encuentren su espacio en los medios de comunicación y lidien con los distintos poderes y grupos de interés $(\text { politics })^{2}$.

Este Monográfico arranca con los dos factores de riesgo mitigables que más han perjudicado y perjudican la salud de las poblaciones, especialmente la de los países desarrollados: el tabaquismo y la obesidad. Para el enfoque económico ni la adicción tabáquica ni la obesidad son males absolutos, de ahí que haya que ofrecer argumentos para la regulación, o el marketing social, de unos ámbitos con múltiples intereses en juego. Estos argumentos se desarrollan tanto para el tabaquismo como para la obesidad y en ambos casos se revisa también lo conocido sobre la efectividad de las políticas dirigidas a mejorar el bienestar social actuando sobre esos dos factores de riesgo.

Ángel López, economista, y Arántzazu Viudes, pediatra, fundamentan las intervenciones públicas sobre el tabaquismo en base a dos familias de fallos de mercado y revisan la efectividad de dichas intervenciones para finalizar con un corolario para España, tan preciso como fundamentado ${ }^{3}$. Las dos familias de fallos de mercado -los efectos del fumar sobre otras personas y los fallos en la racionalidad de los individuos- provocan unas intervenciones, claramente efectivas, dirigidas a los mecanismos que provocan los fallos.

El conocimiento disponible revela que las externalidades financieras no justifican un nivel impositivo mayor al ya aplicado en muchos países desarrollados. No obstante, se precisa reforzar los mecanismos que proporcionan mayor control de las personas 
sobre su vida, cuando tratan de decidir si empiezan a fumar, dejan de fumar o mantienen el empeño de abandonar la adicción.

Las restricciones al consumo en lugares públicos cerrados y unos precios más altos constituyen algunos de estos mecanismos que, con suave paternalismo, posibilitan a las personas la recuperación de las riendas de su trayectoria vital. En España, la política anti-tabáquica se viste de largo en 2005 con la Ley 28 y, en 2006, pone freno a la proliferación de marcas por debajo de 2 euros. No acaba de funcionar, en cambio, la regulación en la hostelería, donde las normas sociales implícitas favorecen el que la mayoría de los locales permitan fumar; los autores consideran recomendable la prohibición total. En lo que respecta a la cuestión, a dirimir empíricamente, del sentido del impacto sobre el bienestar social de una subida del nivel impositivo, López y Viudes, respaldan la estimación experta que apunta a unos beneficios mayores que las pérdidas en el placer de fumar, derivada de una subida impositiva que, para España, pasaría por alcanzar lo antes posible el nivel de los 90 euros por 1000 cigarrillos que la Directiva Europea sugiere para 2014.

Alex Rodríguez y Beatriz González, ambos de la Universidad de Las Palmas de Gran Canaria, analizan la obesidad, creciente entre las personas adultas españolas y con un gradiente social mayor que en los países de nuestro entorno, particularmente para las mujeres ${ }^{4}$. La pobreza y el nivel bajo de educación académica se asocian claramente con la obesidad, aunque conviene saber mejor cuál de los dos tiene más fuerza causal, pues para nada serviría actuar sobre precios si las pautas de consumo vienen determinadas por la educación.

Existe un amplio debate sobre atribución de morbilidad a la obesidad que no alterará su importancia como problema de salud pública cuyos determinantes históricos parecen radicar en la economía (los precios de los alimentos obesogénicos han bajado; frutas y verduras frescas al alza; mayor coste de oportunidad del tiempo de las mujeres con su incorporación al mercado laboral) y en la tecnología (que ha provocado un descenso del consumo calórico).

Rodríguez y González revisan los fundamentos de la regulación social de la obesidad destacando, como López y Viudes para el caso del tabaquismo, las limitaciones a la racionalidad que cercenan la soberanía del consumidor y que llevan a la consideración de la salud como bien tutelar que ha de ser protegido. De nuevo, como con el caso del tabaquismo, esta voluntad social de influir favorablemente en las preferencias de los ciudadanos gana legitimidad por la eventual irreversibilidad de la obesidad, por adictiva.

En el artículo se esbozan los hallazgos de causación genética de la obesidad y se mencionan los recientemente descubiertos fenómenos de interacción social -a los que uno de los dos autores ha contribuido destacadamente $^{5}$ - que establecen el carácter 'contagioso' de la obesidad, a través de redes sociales, en una comunidad progresivamente más corpulenta, que comparte estilos de vida y otras influencias ambientales. Dado que la epidemia crece en redes sociales y que la obesidad es una causa (y efecto) de la pobreza, las políticas contra la obesidad son en último término políticas sociales pro-igualdad.

En relación a la efectividad y coste-efectividad de las intervenciones se sabe más de las individuales que de las comunitarias, por las razones que el artículo expone. Entre las actuaciones individuales, los medicamentos tienen una eficacia muy modesta y sólo en indicaciones muy seleccionadas resultan financiables públicamente. La cirugía, con todos sus riesgos, es la opción más coste-efectiva para las personas con obesidad mórbida. En cuanto a las intervenciones comunitarias, también exis- 
ten efectividades documentadas para algunas, como la de regular la publicidad de TV dirigida a niños, pero de menor aplicación directa al entorno español por problemas de validez externa.

Particular interés reviste un abordaje, como el del National Institute for Health and Clinical Excellence, que integra las guías clínicas y de salud pública, individualiza los mensajes al tiempo que los contextualiza y hace de la actuación sobre la obesidad una política social. Hay que aprender, pues, de los que van por delante pero obteniendo, como reclaman Rodríguez y González, conocimiento sobre el coste-efectividad de las intervenciones en España, particularmente de las comunitarias.

Tras los dos artículos anteriores, con referencias compartidas a la racionalidad limitada, como fundamento de un paternalismo suave, o asimétrico como se le denominará en esta tercera colaboración especial, encajaba bien un panorama exhaustivo acerca de lo que se conoce sobre los límites a la racionalidad. José-Ramón Repullo, salubrista con querencias tempranas por la Psiquiatría, extiende aquí ${ }^{6}$ un trabajo que presentó en las XXVIII Jornadas de Economía de la Salud, celebradas el 2008 en Salamanca. La mente humana evolucionó durante miles de años y en la actualidad presenta dos grandes inadaptaciones a un entorno que poco tiene que ver con aquél del que partió: la dificultad de cooperar con extraños tras haberse limitado hasta hace poco a la interacción dentro de grupos muy reducidos y las que pueden englobarse bajo el epígrafe de comportamiento irracional.

La irracionalidad predecible, cuyo estudio ya ha sido distinguido con algún premio Nobel, como el de Kahneman, está recibiendo una creciente atención, facilitada por medios más potentes para investigar el cerebro. Lo hasta ahora conocido permite a Repullo una sistematización en cinco fuentes empíricamente constatadas de irraciona- lidad, desde el objeto de la decisión hasta los valores que la informan.

La lectura del artículo de Repullo permite apreciar la pertinencia de los ejemplos y, sobre todo, la riqueza de sugerencias, tan sensatas como prácticas, para conseguir mayor efectividad en las políticas de salud. Que ante elecciones relativamente complejas los individuos tengamos configurada por defecto la alternativa más conveniente es una de esas sugerencias, una que precisamente las empresas de tarjetas de crédito prefirieron no adoptar.

Sin sonido de trompeta, con el artículo de Puig-Junoy y Peiró ${ }^{7}$, tan conceptual y riguroso como cotidiano, se cambia el tercio para tomar los 'trastos' de evaluación económica que pueden permitir, aquí y ahora, contribuir a mejorar la deseabilidad de nuestro sistema sanitario, la mejor garantía de su sostenibilidad. Se establece una diáfana distinción entre: 1/ la utilidad terapéutica, propia de la perspectiva clínico-farmacológica, adecuada para las decisiones sobre autorización de medicamentos; 2/ el valor terapéutico añadido que amplía el anterior e incluye otros resultados más allá de los clínicos, definiéndose de forma incremental frente a las mejores alternativas existentes, no el placebo; $3 /$ el coste-efectividad incremental o relación entre valor terapéutico añadido y el coste de oportunidad, el más adecuado desde una perspectiva colectiva para decisiones sobre financiación pública de medicamentos y regulación de precios.

Tras reseñar las innovaciones regulatorias en el sistema comparado, el artículo entra a detallar los cambios pendientes en España donde más allá de las declaraciones políticas, el marco regulatorio y la voluntad de los decisores sanitarios no han favorecido hasta el momento el desarrollo de la evaluación económica de medicamentos aplicada a las decisiones de financiación pública de prestaciones sanitarias y a 
las decisiones de precios de medicamentos $y$ de tecnologías médicas. Conviene reducir la opacidad de la regulación farmacéutica y cristalizar el convencimiento de bastantes actores del sistema sanitario para rematar la faena e introducir la evaluación del costeefectividad incremental, inspirándose en las mejores prácticas foráneas, para informar las decisiones sobre priorización, precios y financiación. Incluso, algún día, será imaginable la hasta ahora nunca contemplada negociación sobre la distribución de los beneficios derivados de la auténtica innovación: entre sus generadores y sus financiadores.

Unos precios de inputs sanitarios, basados en el valor añadido, son útiles tanto a la política sanitaria como a la industrial en la medida que refuerzan señales que tratan de estimular la investigación conducente a la innovación socialmente útil, la cual no siempre coincide con aquella que suscita amplia disposición (individual) a pagar en ámbitos presididos por la asimetría informativa y por los límites a la racionalidad con lo que ya nos hemos topado en las tres primeras colaboraciones especiales.

A continuación, José María Abellán, Fernando Ignacio Sánchez y Jorge Eduardo Martínez, todos de la Universidad de Murcia, brindan, como la colaboración de PuigJunoy y Peiró, un instrumento para la aplicación de la evaluación económica a la mejora de las decisiones de política sanitaria $^{8}$. Se trata de un listado de comprobación para aplicar a estudios observacionales y ensayos clínicos (pragmáticos o controlados) que arrojará una puntuación que, por vez primera en España y segunda en el mundo (tras Australia), podría utilizarse, aunque no es imprescindible, para establecer la máxima disposición a pagar, individual, por un año de vida ajustado por calidad en función de la calidad del conocimiento científico sobre su costeefectividad. La checklist, en cualquier caso, tiene vida autónoma para simplemente cuantificar la calidad de la evidencia científica que debería considerarse antes de incorporar una tecnología, tanto en la gestión como en la clínica.

De los instrumentos de evaluación para una política sanitaria más democrática y eficiente, el Monográfico pasa ahora al análisis del impacto del programa Bolsa Familia en Brasil, país de grandes desigualdades en un subcontinente particularmente desigual. Un brasileño, Luis Currais, y dos compañeros suyos de la Universidad de A Coruña, Berta Rivera y Paolo Rungo ${ }^{9}$, revisan el impacto de un programa de transferencia de renta, espoleado por Lula, en la mitigación de la pobreza y la mejora de la salud. Las transferencias del programa Bolsa Familia llegan a la cuarta parte de la población brasileña y están condicionadas, fundamentalmente, a escolarizar y vacunar los niños.

El programa Bolsa Familia ha reducido la gravedad de la pobreza y atenuado la desigualdad en renta. Buena noticia que refuerza la reciente emergencia de las clases medias en Brasil. Entre las personas afectadas ha mejorado la ingesta, aunque no su calidad. Tampoco parecen haber variado los estilos de vida relacionados con la salud, ni siquiera aquéllos de cumplimiento requerido como las vacunaciones. El programa tiene, sin embargo, efecto favorable sobre la educación, importante variable para interrumpir la transmisión dinástica de la pobreza, especialmente cuando la mejora educativa afecta a las niñas.

Los objetivos relacionados con la reducción de la malnutrición han tenido un cumplimiento parcial a corto plazo. Más parcial ha sido el cumplimiento de objetivos relacionados con la salud que no va mucho más allá de conseguir una mayor frecuentación de las mujeres. Los autores sugieren razones muy plausibles para ello: desde una oferta sanitaria poco elástica y escasamente 
accesible hasta la presencia de incentivos económicos para escolarizar (aunque lo importante, en todo el mundo, más que la escuela a la que se vaya sea el maestro que se tenga) que no se dan, por ejemplo, para vacunar a los niños.

Los resultados del artículo de Rivera, Currais y Lungo contribuyen a un debate sobre el que no existen conclusiones diáfanas. Renta y salud han estado históricamente correlacionadas y resulta obvio que no cabe la segunda sin la primera. Los mecanismos de conexión entre renta $\mathrm{y}$ salud resisten, sin embargo, una explicación simple. Se discute si en estas últimas décadas las mejoras de la salud no habrán venido tan motivadas por la renta como por la educación, la calidad de las instituciones o las prioridades políticas de un país ${ }^{10}$. Cuesta asimismo descartar causalidades comunes a renta y salud. En cualquier caso, está claro que su mejora sólo podrá derivarse del conocimiento detallado de la realidad brasileña, del funcionamiento del programa Bolsa Familia y del análisis de sus efectos.

El broche la sección de colaboraciones especiales de este Monográfico corre a cargo de Guillem López-Casasnovas, presidente de la International Health Economics Association, quien en un exigente artículo repasa los avances en la disciplina y los conecta con bastantes de los problemas de nuestro sistema sanitario (y de otros, claro) que esperan beneficiarse de una solvente aplicación del análisis económico ${ }^{11}$.

Empieza considerando la Economía de la Salud como aquello que hacen los economistas, en la definición atribuida a Jacob Viner y empleada con cierta frecuencia cuando se analiza la evolución de la disciplina $^{12}$, para señalar que un buen economista economista debe contemplar tanto gastos como ingresos, conocer el impacto en el bienestar de las distorsiones impositivas y no confundir la sociedad con el Estado.
López-Casasnovas se muestra partidario de evaluar favorablemente las medidas de política sanitaria con un valor actual neto positivo (diferencia entre beneficios y costes descontados al momento presente), lo cual preludia una jugosa caracterización, incluso sociológica, de welfaristas y nowelfaristas que estimulará la curiosidad del lector. Ambas son dos ramas de la Economía normativa, la que se pronuncia sobre lo que debería ser, las cuales difieren en muchos aspectos ${ }^{13}$ injustamente resumibles diciendo que: 1. Los welfaristas tratan de maximizar una cierta agregación de utilidades buscando la satisfacción de las preferencias individuales con el criterio de que quiénes ganan como consecuencia de medidas de política puedan compensar a los que pierden. 2. Los no-welfaristas tratan de maximizar una agregación de las medidas subjetivas de salud. Existen intentos de conciliar ambos enfoques y críticas solventes a la Economía normativa en general ${ }^{14}$ como la del Nobel Amartya Sen. Hay que procurar compartir el sentido de los términos empleados (no siempre coincide lo técnico con lo coloquial) y explicitar valores (sabiendo incluso que de algunos no somos conscientes), porque la función de bienestar social que se pretenda maximizar ha de poseer la legitimidad que sólo los procesos democráticos participativos y el consenso sobre criterios otorgan, más allá de la eficiencia.

El artículo de López-Casasnovas, tras mencionar someramente la relevancia de los temas abordados por la Economía de la Salud -con unas bases disciplinarias asentadas, que ha contribuido a reforzar, y con una potencia de fuego muy mejorada- finaliza acometiendo contra las barreras construidas por los sicofantes intelectuales del statu quo para excluir de la indagación científica y, sobre todo, del debate público cuestiones definitorias de nuestra evolución inmediata. Temas como la financiación privada, que puede encauzar una parte de la fortísima disposición a pagar por servicios 
de salud, o la eficiencia comparativa de distintas formas organizativas (alternativas a la burocratización), así como el del enrocamiento de intereses particulares para extraer canonjías públicas no visualizables como 'lucro', o el del papel de la responsabilidad individual y la consideración de la efectividad de los servicios para delimitar la cartera de servicios, esperan ser investigados.

La sección de Originales del Monográfico recoge tres artículos. El primero corresponde a los valencianos Peiró y Meneu y al aragonés Bernal. La pedagogía implícita en Variabilidad, desigualdad y efectividad... ${ }^{15}$ permite utilizar este artículo científico como apólogo (una fábula protagonizada por personas y la cirugía oncológica de sus úteros y sus próstatas) para insistir en que más no siempre es mejor y añadir que, en ocasiones, pueden existir desigualdades saludables si una menor utilización protege de procedimientos de utilidad dudosa. Si hace tiempo quedó claro que el camino a la eficiencia pasa por la efectividad, ahora podrá añadirse que la efectividad de los procedimientos condiciona la consecución de la equidad y que, en ocasiones, una menor utilización protege la salud y resulta socialmente admisible desde cualquier perspectiva.

Juan Oliva de la Universidad de Castilla la Mancha, segundo artículo de la sección de Originales, estima el coste social de las pérdidas de productividad laboral que ocasionaron los fallecimientos prematuros en 2005, con datos de la Encuesta de Población Activa y del Registro de Defunciones, tomando la ganancia salarial como indicador de productividad ${ }^{16}$. Las pérdidas de productividad laboral ascendieron al equivalente del 1\% del Producto Interior Bruto, siendo las causas externas y los tumores responsables de la mitad de dichas pérdidas. La causa de muerte con mayor impacto en España, tanto en productividad como en años potenciales de vida perdidos o años potenciales de vida laboral perdidos, son los accidentes de circulación, seguidos del tumor maligno de tráquea, bronquios y pulmón. El descenso de accidentes posterior al 2005, fruto posiblemente de una acción transversal sobre la salud (viaria, educativa...), podría tener refrendo de efectividad con un análisis como el de este artículo de Oliva. Estamos, pues, ante otro método para ayudar a seleccionar actuaciones sobre la salud.

El último artículo de la sección de Originales realiza una correlación transversal entre la carga de enfermedad y la financiación de la investigación en el SNS. Catalá, Álvarez, Génova y Morant, de la Escuela Nacional de Sanidad, documentan una asociación positiva, más bien alta, entre la financiación pública de la investigación sanitaria y los años de vida ajustados por discapacidad para 21 categorías de enfermedades $^{17}$.

Gracias a todos los que acudieron a la convocatoria de este Monográfico y a quienes revisaron los artículos remitidos, así como a Cristina Pérez Andrés, del comité de redacción de la RESP, por contar para este monográfico con mi colaboración.

\section{BIBLIOGRAFÍA}

1. Ortún V, Meneu R. Impacto de la Economía de la Salud en la Política y Gestión Sanitaria. Rev Esp Salud Pública 2006; 80: 491-504.

2. Hernández-Aguado I, Fernández-Cano P. Políticas de salud y salud pública. Gac Sanit 2007; 21(4): 280-81.

3. López A, Viudes A. El control del tabaquismo desde la perspectiva de la economía. Rev Esp Salud Pública 2009; 83(1): 9-24.

4. Rodríguez A, Lopez-Valcarcel BG. El trasfondo económico de las intervenciones sanitarias en la prevención de la obesidad. Rev Esp Salud Pública 2009; 83(1): 25-42.

5. Harris JE, Lopez-Valcarcel BG. Asymetric social interaction in economics: cigarette smoking 
among Young people in the United States, 19921999. J. Health Econ. 2008; 27(2): 249-264.

6. Repullo JR. Identificación y modulación del comportamiento sistemáticamente irracional en medicina y salud pública. Rev Esp Salud Pública 2009; 83(1): 43-58.

7. Puig-Junoy J, Peiró S. De la utilidad de los medicamentos al valor terapéutico añadido y a la relación coste-efectividad incremental. Rev Esp Salud Pública 2009; 83(1): 59-70.

8. Abellán JM, Sánchez FI, Martínez JE. La medición de la calidad de los estudios de evaluación económica. Propuesta de 'checklist' para la toma de decisiones. Rev Esp Salud Pública 2009; 83(1): 71-84.

9. Rivera B, Currais L, Rungo P. Impacto de los programas de transferencia condicionada de renta sobre el estado de salud: el programa bolsa familia de Brasil. Rev Esp Salud Pública 2009; 83(1): 85-98.

10. Cutler D, Deaton A, Lleras-Muney A. The determinants of mortality. J Econ Perspect. 2006; 29: 97-120.

11. López-Casasnovas G. Treinta años de evolución de la economía de la salud. Rev Esp Salud Pública 2009; 83(1): 99-108.
12. Emerging economists. International bright young things. The Economist 30 diciembre 2008.

13. Hurley J. An overview of the normative economics of the health sector. En: Culyer A, Newhouse J: Handbook of Health Economics. Amsterdam: North-Holland Elsevier, 2000, capítulo II.

14. Sen A. Personal utilities and public judgment: or what's wrong with welfare economics. Economic Journal 1979; 89: 537-58.

15. Peiró S, Meneu R, Bernal E. Variabilidad, efectividad y desigualdad. Hsterectomías y prostatecto mías por enfermedad neoplásica en España. (2002-2004) Rev Esp Salud Pública 2009: 83 (1):109-122.

16. Oliva J. Pérdidas de producción laboral ocasionadas por los fallecimientos prematuros en España en el año 2005. Rev Esp Salud Pública 2009; 83(1): 123-136

17. Catalá F, Álvarez E, Génova R, Morant C. Relación en España entre la investigación financiada por Sistema Nacional de Salud y la carga de enfermedad en la comunidad. Rev Esp Salud Pública 2009; 83(1): 137-152. 
